\title{
Evaluación del salto de jugadores elite de voleibol mediante dos métodos: ecuaciones de potencia de salto y plataforma de fuerza
}

\author{
C. Hertogh, O. Hue \\ $\mathrm{J}$ Sports Medicine and Physical \\ Fitness 2002; 42: 300-303 \\ Volumen 42 , No. 3
}

\section{Resumen}

Palabras clave: Ejercicio, fisiología del músculo esquelético, fisiología de la actividad motora.

\section{Abstract}

Antecedentes: El objetivo del presente estudio fue determinar la mejor ecuación de potencia de salto en la evaluación de jugadores elite de voleibol mediante la plataforma de fuerza y las ecuaciones de potencia pico.

Métodos: Nueve jugadores elite de voleibol y nueve sujetos sedentarios ejecutaron pruebas de movimiento contrario sobre una plataforma.

Resultados: La potencia pico y la altura fueron mayores en los jugadores de voleibol que en los sujetos sedentarios, independientemente del método usado.

Los resultados mostraron una correlación entre los valores de potencia pico obtenidos en la plataforma de fuerza y los calculados con las ecuaciones de Lewis, Harman y otros, y Sayers y otros cuando se tomaba en cuenta toda la muestra. Sin embargo, una significativa interacción por nivel de las ecuaciones $\left(\mathrm{p}<10-^{-4}\right)$ indicó diferentes conductas en función del nivel de ejecución.

En los sujetos sedentarios, la potencia pico fue significativamente subestimada al usar la ecuación de Lewis (943 $\pm 162 \mathrm{~W} ; \mathrm{p}<$ $\left.10-{ }^{4}\right)$ y no difería con las ecuaciones de Harman $(3004 \pm 563 \mathrm{~W}$ ) y de Sayers $(3004 \pm 604 \mathrm{~W}$ ) al compararlas con la potencia pico registrada con la plataforma de fuerza $(3372 \pm 532 \mathrm{~W})$.

Por el contrario, en los jugadores de voleibol la potencia pico fue subestimada al usar las tres ecuaciones $\left(1246 \pm 78 \mathrm{~W}, \mathrm{p}<10--^{4}\right.$; $4314 \pm 216 \mathrm{~W}, \mathrm{p}<0,001$ y $4607 \pm 251, \mathrm{p}<0,005$ para las ecuaciones de Lewis, Harman y Sayers respectivamente, frente a $5355 \pm$ $522 \mathrm{~W}$ para la plataforma de fuerza).

Conclusiones: Los resultados del presente estudio demuestran la dificultad para escoger la ecuación más relevante en el cálculo de la potencia de salto.

Background. The aim of the present study was to determine the best jump power equation in the evaluation of elite volleyball players using both the force platform and peak power equations.

Methods. Nine elite volleyball players and nine sedentary subjects performed counter-movement jump tests on a force platform. Results. Peak power and height were greater in the volleyball players than in the sedentary subjects, whatever the method used.

The results demonstrated that the peak power values obtained on the force platform and those scored from the equations of Lewis, Harman and Sayers et al. were correlated when the whole sample was taken into account. However, a significant equation $x$ level interaction $\left(\mathrm{p}<10^{-4}\right)$ indicated different behaviour as a function of performance level. In sedentary subjects, peak power was significantly underestimated using The Lewis equation ( $\left.943 \pm 162 \mathrm{~W} ; \mathrm{p}<10^{-4}\right)$ and did not differ using both the Harman (3004 \pm 563 W) and Sayers $(3400 \pm 604 \mathrm{~W})$ equations when compared to the peak power noted with the force platform $(3372 \pm 532 \mathrm{~W})$. In contrast, in volleyball players, peak power was underestimated using the three equations ( $1246 \pm 78 \mathrm{~W}, \mathrm{p}<10^{-4} ; 4314 \pm 216 \mathrm{~W}, \mathrm{p}<0.001$; $4607 \pm 251, \mathrm{p}<0.005)$ for the Lewis, Harman and Sayers equations, respectively, versus $5355 \pm 522 \mathrm{~W}$ for the force platform). Conclusions. The results of the present study demonstrate the difficulty in choosing the most relevant equation in the jump power calculation.

Key words: $\quad$ Exercise, physiology - Muscle, skeletal, physiology - Motor activity.

\footnotetext{
1 Traducción de Daniel Ignacio Oliveros Wilches, profesor de la Universidad Pedagógica Nacional. Tomado de: Laboratoire ACTE,
} UFR-STAPS, Université des Antilles et de la Guyane. France (Guadeloupe). 
La potencia de ejecución es un componente esencial para obtener buenos resultados en muchos deportes. En el caso de los jugadores de voleibol, se recomiendan los ejercicios orientados al aumento de la fuerza para mejorar la potencia de ejecución y, por lo tanto, la máxima altura de salto (Bosco et al., 1982; Young, Wilson y Byrne, 1999; Clutch et al., 1983).

Recientemente, en una comparación de diferentes métodos para analizar la ejecución del salto con caída, Baca (1999) demostró que los métodos que usaban la plataforma de fuerza eran más precisos. Sin embargo, el uso de la plataforma para determinar la potencia de salto vertical es prohibitivo y no siempre está al alcance de los recursos financieros de un club de voleibol. Más aún, la plataforma no es el método perfecto y puede haber circunstancias en las cuales sea preferible usar las ecuaciones: Newton et al. (1999) sugieren que los métodos de plataforma no reflejan las mejoras derivadas del entrenamiento tan bien como los test de saltar y alcanzar; más aún, consideran que los métodos de medición con plataforma pueden restringir la técnica en deportistas expertos (en este caso jugadores de voleibol). Por estas razones, es esencial contar con un método práctico y económico para valorar la potencia humana de ejecución.

En 1974, se desarrolló la fórmula de Lewis para calcular la potencia de ejecución de un deportista a partir del peso corporal y la distancia de salto vertical (Lewis, 1981), que ha sido ampliamente usada tanto en fisiología del ejercicio como en pruebas y mediciones de investigación. Sin embargo, hace poco fue controvertida; primero por Harman et al. (1991), y luego por Sayers et al. (1999). Este último grupo observó que su ecuación puede usarse para determinar la potencia pico, en lugar de las fórmulas de Harman et al. y de Lewis. No obstante, hasta donde se sabe, estas ecuaciones nunca se han usado con jugadores profesionales de voleibol.

El objetivo del presente estudio fue entonces determinar si las mismas ecuaciones son apropiadas tanto para deportistas de elite masculinos (en este caso jugadores competitivos de voleibol) como para hombres que no practican ningún deporte. Se usaron dos métodos de evaluación del salto: la plataforma de fuerza y el test de saltar y alcanzar.

\begin{tabular}{c|c|cc|c|}
\hline Grupo & No. & Edad (años) & Masa corporal (kg) & Estatura (cm) \\
\hline G (Todos) & 18 & $21,7 \pm 2,9$ & $75,7 \pm 7,8$ & $182,8 \pm 7,4$ \\
G1 (Deportistas) & 9 & $21,2 \pm 3,0$ & $78,5 \pm 4,7$ & $185,7 \pm 6,0$ \\
G2 (Sedentarios) & 9 & $22,2 \pm 3,1$ & $72,8 \pm 7,9$ & $180,0 \pm 7,8$
\end{tabular}

Tabla 1. Características antropométricas de los participantes en el estudio.

\section{Materiales y métodos}

\section{Sujetos}

En el presente estudio participaron 18 hombres saludables, con edades entre 18 y 26 años, que fueron separados en dos grupos de acuerdo con sus hábitos deportivos. El grupo $G_{1}$ lo conformaron 9 jugadores profesionales de voleibol de una liga francesa del escalafón nacional, y el grupo $G_{2} 9$ estudiantes universitarios que no realizan ninguna actividad deportiva. Se estableció correspondencia entre ambos grupos de acuerdo con la edad, la masa corporal y la estatura. Todos los sujetos estaban familiarizados con el test de salto vertical. Las características antropométricas y las actividades deportivas se relacionan en la tabla 1. Todos los sujetos fueron informados del propósito de este estudio, según lo ordenado por el Comité de ética de la Universidad.

Formula de Lewis:
Potencia $\frac{(\mathrm{kg} \times \mathrm{m})}{\mathrm{s}}=\sqrt{4,9 \times \text { masa corporal }(\mathrm{kg}) \times \mathrm{h}(\mathrm{m})}$
Se multiplica por 9,8 para obtener watts
$\mathrm{h}=$ resultado de saltar $\mathrm{y}$ alcanzar

Formula de Harman et al:?

Potencia pico $(\mathrm{W})=61,9 \times \mathrm{h}(\mathrm{cm})+36,0 \times$ masa corporal $(\mathrm{kg})-1822$

Formula de Sayers ot al 3 :

Potencia pico $(\mathrm{W})=51,9 \times \mathrm{SMC}(\mathrm{cm})+48,9 \times$ masa corporal (kg) - 2007.

$\mathrm{SMC}=$ Salto con movimiento contrario

Tabla 2. Ecuaciones de potencia de salto usadas en el presente estudio

\section{Plataforma de fuerza}

Se ejecutaron saltos con movimiento contrario (SMC) en una plataforma experimental de fuerza $(555 \times 555$ $\mathrm{mm}$ ) con galgas de deformación (Newton et al., 1999). La plataforma podía medir hasta $8.000 \mathrm{~N}$ de fuerza vertical. Los errores obtenidos fueron del $1 \%$ para los componentes verticales y menos del $4 \%$ para los horizontales. La interferencia de los componentes de deformación fue del $1 \%$. Las fuerzas verticales sobre la plataforma generaron señales eléctricas que fueron convertidas por un convertidor de corriente análoga a digital (PC.LAB). El software experimental, desarrollado con el laboratorio Inserm U.103 (Newton et al., 1999), registró y alma- 
cenó los datos y las curvas generados. Se ejecutaron tres saltos con intervalos de 5 minutos de descanso, y se usó el mejor salto para el análisis.

La potencia de salto se calculó para la plataforma y para las ecuaciones de Lewis (1981), Harman et al. (1991), y Sayers et al. (1999) (tabla 2), introduciendo las variables de altura de salto y peso corporal.

\section{Análisis estadístico}

Las variables obtenidas con la prueba de plataforma de fuerza y con cada fórmula (Lewis, 1981; Harman et al., 1991, y Sayers et al., 1999) se analizaron usando el análisis de doble varianza (método $\times$ grupo). Se calcularon los coeficientes de correlación entre todas las variables usando los programas Systat. La significancia estadística fue aceptada en el nivel $p<0,05$.

\begin{tabular}{|c|c|c|c|c|}
\hline Grupo & $\begin{array}{l}\text { "optencla en } \\
\text { la platarorna } \\
\text { W }\end{array}$ & \multicolumn{2}{|c|}{ Potencia Ersedicha Wh } & 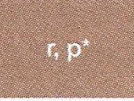 \\
\hline G (Todos) & $4.364 \pm 1141$ & $\begin{array}{l}\text { Lewis } \\
\text { Harman } \\
\text { Sayers }\end{array}$ & $\begin{array}{l}1094 \pm 199^{* *} \\
3659 \pm 791^{* *} \\
4004 \pm 766^{* *}\end{array}$ & $\begin{array}{l}0,63,0,005 \\
0,69,0,001 \\
0,65,0,003\end{array}$ \\
\hline$G_{1}$ (Deportistas) & $5355 \pm 522$ & $\begin{array}{l}\text { Lewis } \\
\text { Harman } \\
\text { Sayers }\end{array}$ & $\begin{array}{c}1246 \pm 78^{* *} \\
4314 \pm 216^{* *} \\
4607 \pm 251^{* *}\end{array}$ & $\begin{array}{l}-0,25, \mathrm{~ns} \\
-0,07, \mathrm{~ns} \\
-0,19, \mathrm{~ns}\end{array}$ \\
\hline $\mathrm{G}_{2}$ (Sedentarios) & $3372 \pm 532$ & $\begin{array}{l}\text { Lewis } \\
\text { Harman } \\
\text { Sayers }\end{array}$ & $\begin{array}{c}943 \pm 162^{\star *} \\
3004 \pm 563^{* *} \\
3400 \pm 604^{* \star}\end{array}$ & $\begin{array}{l}0,30, \text { ns } \\
0,42, n s \\
0,34, n s\end{array}$ \\
\hline
\end{tabular}

* p: Valores de $p$ obtenidos de la correlación $(r)$ entre la potencia pico medida en la plataforma y la calculada con las ecuaciones.

${ }^{* *}$ Diferencias significativas.

Tabla 3. Comparaciones de la potencia pico medida sobre la plataforma de fuerza con potencia pico predicha con las ecuaciones

\section{Resultados}

\section{Entre métodos}

Los valores de potencia pico obtenidos en la plataforma de fuerza y los registrados a partir de las ecuaciones de Lewis (1981), Harman (1991) y Sayers (1999) mostraron correlaciones significativas cuando se aplicaron a toda la muestra (tabla 3); sin embargo, no mostraron una correlación significativa cuando se aplicaron a $G_{1}$ y $G_{2}$ por separado. Más aún, una significativa interacción por nivel de las ecuaciones $\left(\mathrm{p}<10^{-4}\right)$ demostró un comportamiento diferente en relación con el nivel de ejecución. Al tomar en consideración toda la muestra, la potencia pico fue significativamente subestimada tanto con las ecuaciones de Lewis $\left(\mathrm{p}<10^{-4}\right)$ como con la de Harman $(\mathrm{p}<0,04)$ (tabla 3).
Sin embargo, con la ecuación de Sayers no hubo diferencias significativas con los valores obtenidos en la plataforma de fuerza. En $G_{2}$ la potencia pico fue significativamente subestimada $\left(\mathrm{p}<10^{-4}\right)$ al aplicar la ecuación de Lewis, pero no difirió mucho de la predicha en la plataforma al usar las ecuaciones de Sayers y Harman. Por el contrario, en $\mathrm{G}_{1}$ la potencia pico fue subestimada al usar las tres ecuaciones (tabla 3).

\section{Entre grupos}

La potencia pico fue mayor (tabla 3 ) en los jugadores de voleibol que en los sujetos sedentarios, independientemente de la prueba o ecuación usada $\left(\mathrm{p}<0,002, \mathrm{p}<10^{-4}\right.$, $\mathrm{p}<0,001, \mathrm{p}<10^{-4}$ para las ecuaciones de Lewis, Harman y Sayers y la plataforma de fuerza, respectivamente).

\section{Análisis y conclusiones}

Este estudio demuestra lo difícil que resulta analizar la ejecución del salto con caída, en particular en poblaciones de alto rendimiento.

Recientemente, mientras realizaban una validación cruzada de una nueva ecuación de potencia pico (o máxima), Sayers et al. (1999) mostraron que era más precisa que la tradicional de Lewis (1981). Más aún, sugieren que podría ser considerada de mayor validez que la ecuación de Harman et al. (1991), ya que ésta se apoya en datos de una población más grande y diversa. Los resultados del presente estudio confirman que para toda la muestra: La ecuación de Sayers es la única cuyo valor medio de potencia pico no fue significativamente diferente de la medida con la plataforma.

Al parecer, los valores obtenidos con las ecuaciones de Harman y Sayers no eran diferentes de los medidos en la plataforma en la población sedentaria. En cambio, los valores obtenidos con las tres ecuaciones fueron significativamente diferentes de los obtenidos en la plataforma de fuerza para jugadores de voleibol elite. Estos resultados pueden explicarse por la diferencia en la técnica del salto con movimiento contrario (SMC, es decir, saltar y alcanzar) en nuestros jugadores de voleibol y sujetos sendentarios. Sayers et al. (1999) consideran que las diferencias en la magnitud y rapidez del movimiento contrario contribuyen al error en la predicción de la potencia pico. Bobbert y Van Soest (1994) demostraron la importancia de la técnica (o sea, del control del movimiento) en la ejecución del salto vertical durante el SMC, y Kovacs et al. (1999) demostraron que la colocación del pie modifica la cinemática y la cinética durante el salto con caída. A pesar de que el grupo sedentario 
estaba familiarizado con el SMC, no se puede descartar la posibilidad de que, con entrenamiento diario, los jugadores de voleibol hayan desarrollado una técnica superior.

Los resultados del presente estudio confirmaron los hallazgos de Sayers et al. (1999) y de Harman y otros (1991) en relación con la potencia pico sustancialmente subestimada que se predijo con la fórmula de Lewis. En la actualidad, esta ecuación se usa ampliamente en la fisiología del ejercicio, ante todo para predecir diferencias de potencia entre sujetos, y entre pruebas en su-

\section{Referencias}

Baca, A. (1999). A comparison of methods for analyzing drop jump performance. Med Sci Sports Exerc 31, 437-442.

Bobbert, W. y Van, Soest A. L. (1994). Effects of muscle strengthening on vertical jump height: a simulation study. Med Sci Sports Exerc 26: 1012-1020.

Bosco, C., Komi, P. V., Pulli, M., Pittera, C. y Montonev, H. (1982). Considerations of the training of the elastic potential of the human skeletal muscle. Volleyball 2, 22-30.

Clutch, D. M.; Wilton, M.; McGown, C. y Bryce GR. (1983). The effect of depth jumps and weight training on leg strength and vertical jump. Res Q Exerc Sport 54, 5-10.

Harman, E. A.; Rosenstein, Nff; Frykman, P. N., Rosenstein, R. M. y Kraemer, W. J. (1991). Estimation of human power output from vertical jump. J Appl. Sports Sci Res 5:116-120.

Hertogh, C.; Micallef, J. y Vaissiere, F. (1991). Test dévaluation de la puissance maximale. Sci Sports (Paris) 6, 185-191. jetos sedentarios y deportistas elite y subelite (White y Johnson, 1991), pero no para predecir valores pico de potencia.

Para concluir, los resultados del presente estudio parecen demostrar la dificultad para escoger la ecuación más adecuada para calcular la potencia de salto en jugadores de voleibol profesionales. Cada método, sin embargo, permitió confirmar la significativa diferencia en la potencia pico entre jugadores de voleibol y sujetos sedentarios, lo cual indica que eran suficientemente precisos para estudiar las diferencias entre nuestros grupos.

Kovacs, I.; Tihanyi, J.; Devita, P.; Racz, L; Barrier, J. y Hortobagyi, T. (1999). Foot placement modifies kinematics and kinetics during drop jumping. Med Sci Sports Exerc 3, 708-716.

Lewis. Citado en: Fox EL, Mathews, M, eds. (1981). The physiological basis of physical education and athletics. Filadelfia: WB Saunders Company: 619-621.

Newton, R.; Kraemer D. y HaIdcinen, K. 1999). Effects of ballistic training on preseason preparation of elite volleyball players. Med Sci Sports Exerc 31, 323-330.

Sayers, S. P. (1999). Harackiewicz, D. V.; Hannan, E. A.; Frykman, P. N.; Rosenstein, W. Cross-validation of three jump power equations. Med Sci Sport Exerc 31, 572-577.

White, A. T. y Johnson, S. C. (1991). Physiological comparison of international, national and regional alpine skiers. Int J Sports Med 12, 374-378.

Young, W. B., Wilson, G. J. y Byrne, C. (1999). A comparison of drop jump training methods: effects on leg extensor strength qualities and jumping performance. Int J Sports Med 20, 295-303. 